\title{
Vitreoretinal junction in infectious endophthalmitis in a primate eye
}

\author{
BENJAMIN MILLER, HEDVA MILLER, AND STEPHEN J RYAN
}

From the Department of Ophthalmology, University of Southern California, and the Estelle Doheny Eye Foundation, Los Angeles, USA

SUMMARY A primate eye affected by postoperative infectious endophthalmitis was studied early in the course of the disease. Retinal perivasculitis, vitreoretinal adhesions along the inflamed vessels, and traction of the partially separated posterior vitreous on the retina at the adhesion sites were observed. It is suggested that such vitreoretinal junction pathology may be responsible for the development of retinal detachment in infectious endophthalmitis.

Endophthalmitis is a devastating complication of intraocular surgery and penetrating eye injury. In spite of modern management, which includes intravitreal injection of antibiotics and steroids, ${ }^{\prime}$ and vitrectomy, ${ }^{23}$ a considerable number of affected eyes are lost.

One of the more ominous sequelae of endophthalmitis is retinal detachment, which is believed to be tractional or exudative $e^{45}$ or rhegmatogenous. ${ }^{6} \mathrm{~A}$ strikingly high incidence of retinal detachment was recently found in a series of cases of post-traumatic endophthalmitis (Jarus G, personal communication, 1984). The exact pathogenesis of the detachment, however, is difficult to prove, since the affected eyes reach the pathologist as disorganised tissue after evisceration or late in the course of the disease as a phthisical eye.

We recently had the opportunity to study a primate eye early in the course of infectious endophthalmitis. We now present the gross and microscopic pathological changes of the vitreoretinal interface of this eye and propose that they may explain the pathogenesis of endophthalmitis-related retinal detachment.

\section{Material and methods}

An adult cynomolgus monkey developed endophthalmitis of the right eye following an unsuccessful attempt to inject gas into the vitreous cavity; all the gas immediately leaked out of the eye through the

Correspondence to Dr S J Ryan, USC Ophthalmology Department, Doheny Eye Foundation, 1355 San Pablo Street, Los Angeles, Ca 90033, USA. injection site. Twenty-four hours later the eye was congested and chemotic, and haziness developed in the cornea, anterior chamber, and vitreous. In spite of intensive topical as well as subconjunctival antibiotic treatment, hypopyon and yellow vitreous exudates followed and within three days obscured the

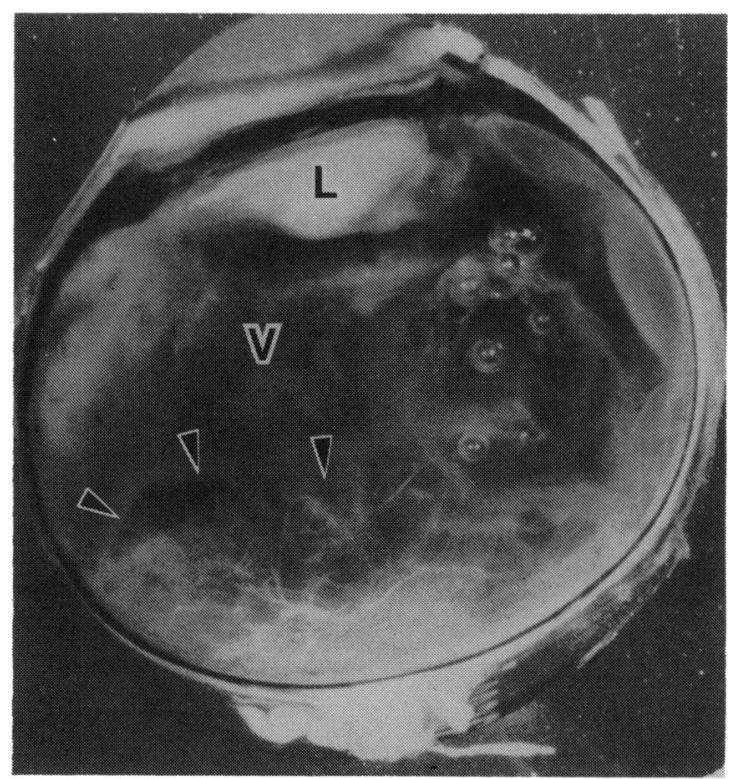

Fig. 1 Right calotte of bisected primate globe affected by infectious endophthalmitis. Opaque vitreous (V) is artefactually separated (arrowheads) from the retina, exposing diffuse retinal perivasculitis. $\mathrm{L}$ indicates lens. 


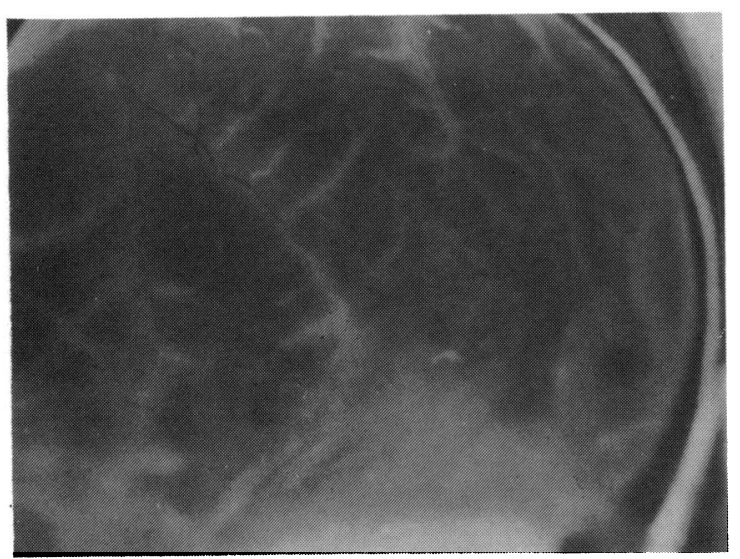

Fig. 2 Dissecting micrograph of right calotte of affected globe showing retinal perivasculitis with extensive exudates and haemorrhages along inflamed vessels.

view of the fundus. The eye was enucleated four days after the first signs of endophthalmitis.

The globe was bisected vertically through the optic nerve, some of the exudate in the vitreous collected, and a smear prepared and stained by Gram's method; the specimen was not cultured. The calottes were immersed in $2 \%$ paraformaldehyde and $2.5 \%$

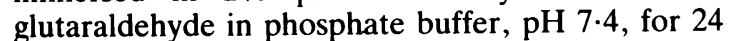
hours and then studied under a dissecting microscope. With a blunt probe the opaque vitreous of the right calotte was separated from the retinal surface for better visualisation of the vitreoretinal border region (Fig. 1). Multiple posterior eye wall blocks were dissected out of the left calotte, dehydrated in graded concentrations of ethanol, embedded in paraffin, sectioned, stained with periodic acid Schiff and haematoxylin-eosin, and studied with a light microscope.

\section{Results}

Dissecting microscopy of the calottes revealed an opaque vitreous containing pockets of dense yellow exudate. Posterior vitreous detachment (PVD) was not present. The smear of the exudate showed Grampositive cocci surrounded by large numbers of polymorphonuclear inflammatory cells. On separating the vitreous from the retinal surface in the right calotte (Fig. 1), a severe perivasculitis was revealed involving the arteries as well as the veins; multiple

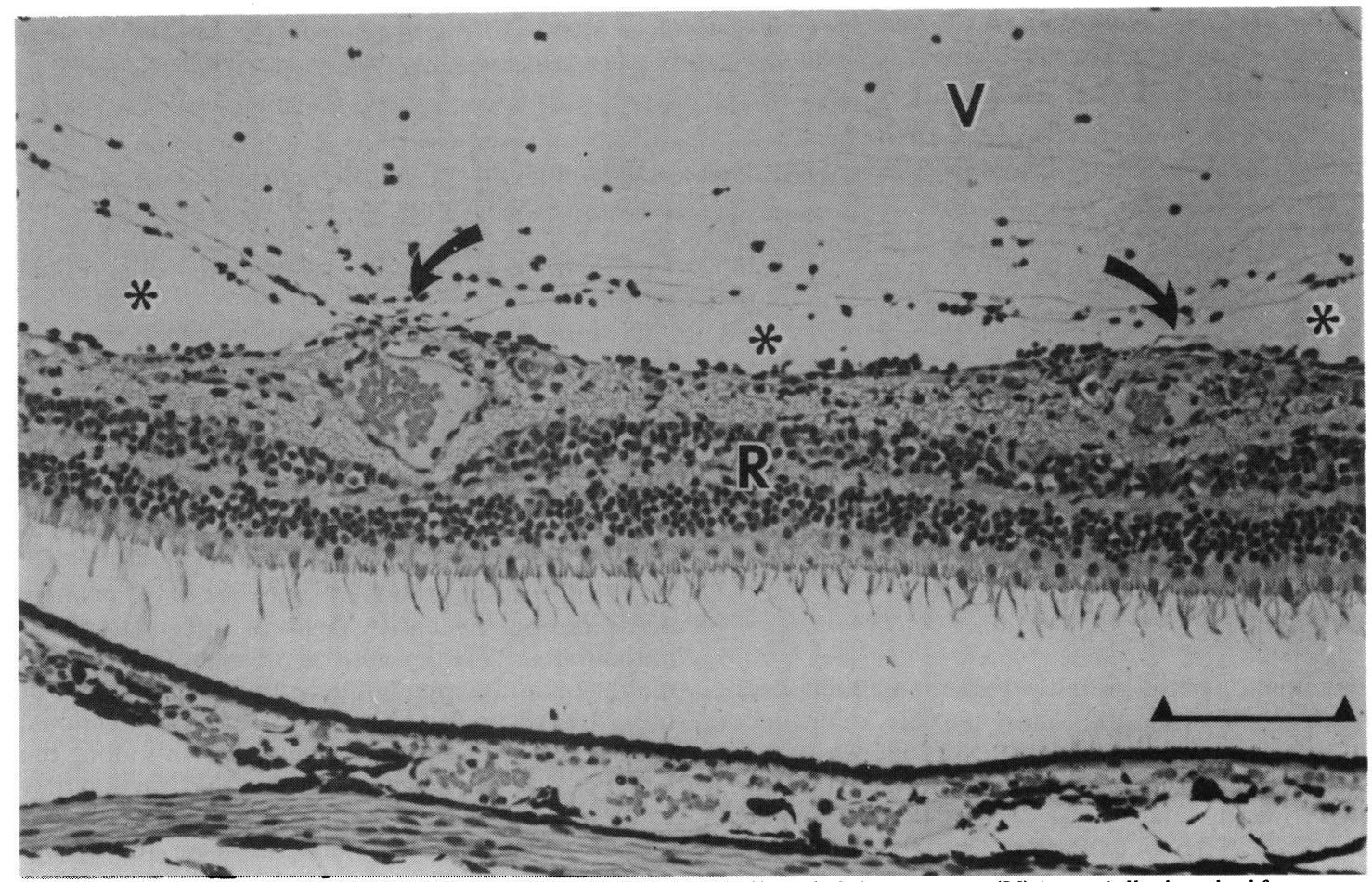

Fig. 3 Light micrograph of vitreoretinal junction in left calotte of affected globe. Vitreous $(\mathrm{V})$ is partially detached from retina $(\mathrm{R})$ and is attached only along inflamed vessels (arrows). Vitreous, subhyaloid space (asterisks), and retina $(\mathrm{R})$ show heavy infiltration by inflammatory cells. Retinal detachment is an artefact. Haematoxylin-eosin, bar $=100 \mu \mathrm{m}$. 


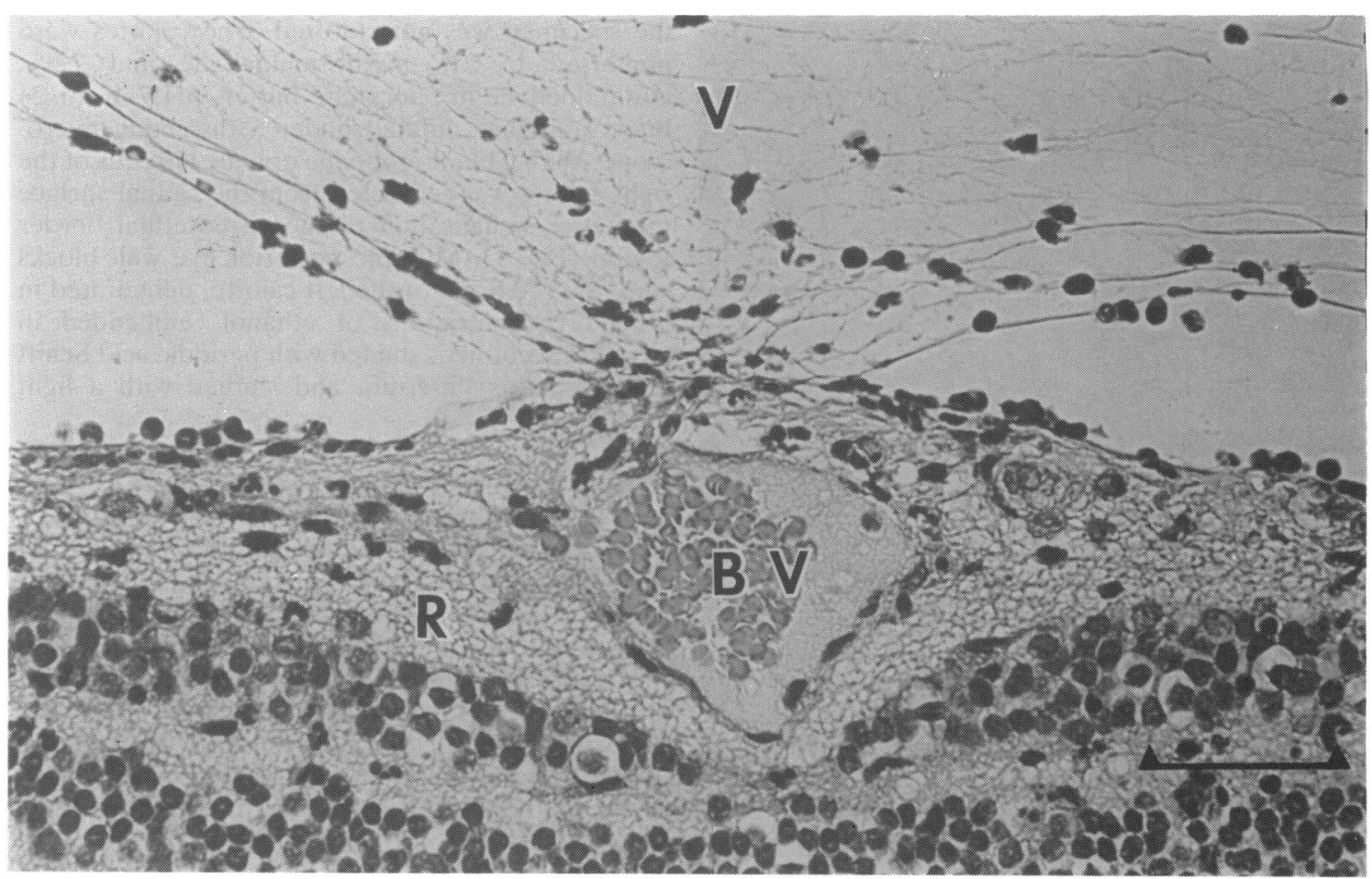

Fig. 4 Light micrograph of vitreoretinal adhesion over inflamed blood vessel (BV). Adherent vitreous (V) is pulling on retina (R). Note polymorphonuclear inflammatory cells infiltrating vitreoretinal border structures and cuffing retinal blood vessels. Haematoxylin-eosin, bar $=50 \mu \mathrm{m}$.

retinal haemorrhages were observed along the affected vessels (Fig. 2).

Light microscopy of the vitreoretinal junction in the left calotte (in which the vitreoretinal relationship was left undisturbed) showed strong adhesions of the vitreous cortex to the retina over the inflamed vessels (Fig. 3). The vitreous appeared to be in the process of separating from the retina; it was partly detached yet still adhering to and exerting tractional forces on the retina overlying the affected vessels. Numerous polymorphonuclear inflammatory cells were seen in the vitreous cortex and throughout the inner retina, particularly cuffing the blood vessels (Fig. 4).

\section{Discussion}

Examination of the primate eye demonstrated severe retinal perivasculitis and partial detachment (microscopic) of the posterior vitreous with strong vitreoretinal adhesions along the affected vessels. These findings were consistent in all the examined tissue blocks, so that an artefact can be excluded. As the injected gas leaked immediately from the eye, it could not have had any possible effect on the vitreous body.
Inflammation within the vitreous is known to produce opacification as well as liquefaction and shrinkage of this tissue, ${ }^{6}$ with resulting PVD. ${ }^{78}$ Endophthalmitis is also associated with retinal periphlebitis. ${ }^{9}$

Posterior vitreous detachment can produce retinal breaks if the outermost vitreous cortex adheres to the retinal surface. ${ }^{10}$ Changes over vessels in the peripheral retina and subsequent pathological vitreoretinal attachments at these sites could explain some of the peripheral retinal holes caused by PVD, "while similar developments may lead to retinal breaks at the posterior retina. ${ }^{12}$

We suggest that a similar sequence of events may occur during the early stages of infectious endophthalmitis. PVD develops as a result of the inflammation and subsequent liquefaction and shrinkage of the vitreous body. This is turn induces tractional forces on the retina at the adhesion points along the inflamed vessels. It is possible that this traction may result ultimately in tractional or rhegmatogenous retinal detachment, but this still needs to be confirmed clinically.

Michels, ${ }^{13}$ describing his technique for vitrectomy in endophthalmitis, states that no effort should be 
made to separate the posterior cortical vitreous from the retina if PVD is not present. His clinical impression is that in many eyes inflammatory debris causes adhesion of the posterior vitreous surface to the inner retina, and any attempt to separate the vitreous may result in tearing of the retina. Our findings support these clinical observations. Furthermore they suggest that the vitreoretinal adhesions may in fact occur in conjunction with the inflamed retinal vessels.

This study was supported by grants EY02061 and EY03040 from the National Eye Institute.

The animal used in this study was maintained in animal care facilities fully accredited by the American Association of Laboratory Animal Science.

\section{References}

1 Peyman GA. Sanders DR. Advances in uveal surgery, vitreous surgery, and the treatment of endophthalmitis. New York: Appleton-Century-Crofts, 1975: 178-228.

2 Eichenbaum DM, Jaffe NS, Clayman HM, Light DS. Pars plana vitrectomy as a primary treatment for acute bacterial endophthalmitis. Am J Ophthalmol 1978; 86: 167-71.
3 Diamond JG. Intravitreal management of endophthalmitis. In: Shimizu K, Oosterhuis JA, eds. Ophthalmology. Amsterdam: Excerpta Medica, 1979: 1571-4.

4 Yanoff M. Fine BS. Ocular pathology: a text and atlas. Philadelphia: Harper and Row, 1982: 81.

5 Hogan MJ, Zimmerman LE. Ophthalmic pathology: an atlas and textbook. 2nd ed. Philadelphia: Saunders, 1962: 549.

6 Hogan MJ. Inflammation and its effect on the vitreous. Trans Ophthalmol Soc UK 1975; 95: 378-81.

7 Streeten BAW. Disorders of the vitreous. In: Garner A. Klintworth GK, eds. Pathobiology of ocular disease: a dynamic approach. New York: Dekker, 1982: 1383-419

8 Chan IM, Jalkh AE. Trempe CL. Tolentino FI. Ultrasonographic findings in endophthalmitis. Ann Ophthalmol 1984; 16: 778-84.

9 Packer AJ, Weingeist TA, Abrams GW. Retinal periphlebitis as an early sign of bacterial endophthalmitis. Am J Ophthalmol 1983; 96: 66-71.

10 Foos RY. Posterior vitreous detachment. Ophthalmology (Rochester) 1972; 76: 480-97.

11 Spencer LM, Foos RY. Paravascular vitreoretinal attachments: role in retinal tears. Arch Ophthalmol 1970; 84: 557-64.

12 Foos RY. Vitreoretinal juncture over retinal vessels. Graefes Arch Clin Exp Ophthalmol 1977; 204: 223-34.

13 Michels RG. Vitreous surgery. St Louis: Mosby, 1981: 345-62.

Accepted for publication 31 July 1986. 